\title{
La evaluación estandarizada en los docentes
}

\begin{abstract}
Mireya Ramos Barba. Universidad Pedagógica Nacional del Estado de Chihuahua, campus Delicias, México. Cuenta con cargo en una Subdirección de Gestión en nivel primaria. Tiene producción relacionada con la evaluación. Correo electrónico: miraba78@ gmail.com. ORCID: https://orcid. org/0000-0002-2736-3173.
\end{abstract}

Guadalupe Iván Martínez Chairez. Escuela Normal Rural Ricardo Flores Magón, Chihuahua, México. Tiene producción académica principalmente en torno a la evaluación del desempeño docente y la formación docente. Correo electrónico: ivan.martinez@ enrrfm.edu.mx. ORCID: https://orcid. org/0000-0001-7925-6305.

\section{Resumen}

La presente investigación se realiza bajo un método cualitativo, con un diseño fenomenológico, usando la técnica de la entrevista, a través de grabaciones sobre anécdotas y experiencias de docentes que han sido evaluados. La pregunta principal de investigación es: ¿Cuáles son las repercusiones de una evaluación estandarizada en el ámbito personal, en la experiencia de los docentes evaluados? Entre los resultados que se encontraron destaca que los profesores consideran que la evaluación fue un instrumento que trató de depurar a la plantilla docente y brindar oportunidad de entrar al servicio a otras profesiones similares a la docencia; la etiqueta que se les asigna con base en el puntaje que se obtiene, punitiva en el sentido de que no respetó las condiciones laborales de quienes fueron llamados a vivir el proceso. Consideran también que solo se tomó en cuenta la parte teórica, no se consideró la práctica, al menos no de manera real, por la facilidad de retocar los productos que se solicitaban a conveniencia propia, al priorizar y dar a conocer solo la parte exitosa de la realidad. Por otro lado, aunque lo que se les pedía era algo inherente a su práctica, enfrentarse a la zozobra de perder su trabajo, su estatus social y económico, cimbró su zona de confort y estabilidad, por lo que se perjudicó de manera significativa su ámbito personal físico, mental y emocional.

Palabras clave: Proceso, evaluación docente, repercusiones.

Abstract
This research is carried out under a qualitative method, with a phenomenological
design, using interview as technique, through recordings of anecdotes and experi-
ences of teachers who have been assessed. The main research question is: What are
the repercussions of a standardized assessment in the personal area, based on the
experience of the teachers who have been assessed? Among the results found stands
out that teachers consider that the assessment was an instrument that sought to purge
the teaching staff and provide opportunities for other teaching related professions to
enter the teaching practice; the label assigned to them based on the score obtained
was punitive, in the sense that it did not respect the working conditions of those
who were called upon to be part of the process. They also consider that only the
theoretical part was taken into account, the practical one was not considered, at least
not realistically, because of the ease of retouching the products that were requested


at their own convenience, by prioritizing and showing only the successful part of the reality. On the other hand, although what was asked of them was something inherent to their practice, having to face the anxiety of losing their job, social or economic status shifted their comfort zone and stability, so their personal environment was significantly damaged, physically, mentally, and emotionally.

Keywords: Process, teacher assessment, repercussions.

\section{INTRODUCCIÓN}

Evaluar a los docentes ha sido un tema controversial en los años recientes. Aunque es una práctica que se lleva a cabo desde hace tiempo, no todos los profesores están dispuestos a transitar por este proceso, algunos piensan que la experiencia en las aulas es más que suficiente, otros que es una profesión que requiere una preparación continua y lo ven como una oportunidad de descubrir qué fortalezas o áreas de oportunidad se tienen para poder incidir en ellas. Para esto, la evaluación tendría que ser formativa, que es de lo que adolece el sistema educativo del país.

Díaz (2019) habla del sentido que debe tener la evaluación, con base en una fundamentación e intención que permita al docente generar la habilidad de reconocer las necesidades que se presentan en su entorno educativo y poder incidir positivamente en ellas, que sus acciones se orienten en tener un conocimiento continuo sobre lo que se evalúa y las acciones pertinentes para generar una retroalimentación que se encamine hacia la búsqueda incesante de brindar una calidad educativa. También en esta sintonía, Cruz, Hincapié y Rodríguez establecen las siguientes características que debe poseer una evaluación docente exitosa:

Primero, definir qué es lo que se espera de un docente de excelencia. Segundo, precisar cuál es el objetivo que busca alcanzar la evaluación docente. Tercero, producir resultados confiables y válidos. Cuarto, usar de manera eficiente los resultados según los objetivos establecidos. Quinto, realizar una investigación continua de los procesos y resultados para retroalimentar el sistema de evaluación [Cruz, Hincapié y Rodríguez, 2020, p. 42].

A partir de que se implementó el Nuevo Modelo Educativo en México, en el año 2017, surgió la necesidad de transformar la realidad de la sociedad a través de una educación que sea para toda la vida, con la consigna de aprender a aprender, continua actualización, adaptación y aprendizaje constante; todo ello con la finalidad de brindar un servicio educativo relacionado directamente con una enseñanza de calidad y, por supuesto, abatir el rezago educativo. Es así como se desarrolló la Nueva Escuela Mexicana (NEM), un plan de 23 años que pretende reforzar al sistema en sus diferentes niveles para individuos de los cero a los 23 años (SEP, 2019).

La perspectiva de esta NEM, que asume a la educación desde el humanismo y a los sujetos partícipes del hecho como prioridad, propone algunas líneas de acción 
permanentes para llevar a cabo esta visión. El primer punto habla de la revalorización del magisterio a través de la reforma de esquemas de actualización y capacitación, reestructuración en la organización de la planta docente y directiva, infraestructura necesaria, generar visiones y proyectos en común, además de atender "mecanismos de estímulo y reconocimiento, mejores condiciones laborales e incremento salarial, así como el adecuado acompañamiento a las maestras y los maestros durante su proceso de retiro" (SEP, 2019, p. 11).

Por lo tanto, se expresa también que "la revalorización es la reivindicación del papel del cuerpo docente como garante de la comprensión, conservación y transmisión del conocimiento, la ciencia y los mejores valores de la sociedad compleja, en permanente cambio e inmersa en crecientes niveles de incertidumbre" (SEP, 2019, p. 12), esto es, se invierten esfuerzos en profesionalizar a los maestros, para que esta preparación tenga como consecuencia subir el nivel académico de los alumnos y baje el rezago en todo el país.

Con base en lo anterior, hay mucho que trabajar para aplicar una evaluación a los profesores con éxito, y que su funcionalidad y efectividad no se reduzca a tomar en cuenta solo aspectos teóricos, más bien llegar a la práctica misma, que es el lugar en el que se expresa la realidad de la sociedad en general. Es por esta razón que, con la tarea de dar voz a la experiencia de quienes entraron a este proceso, se lleva a cabo el siguiente trabajo, cuyo objetivo principal es analizar las repercusiones que han tenido los docentes en el ámbito personal al someterse a una evaluación estandarizada de manera obligatoria.

\section{DISEÑO METODOLÓGICO}

La metodología que guió este estudio fue a través de un paradigma interpretativo, porque "los individuos buscan la comprensión del mundo en el cual viven y trabajan. Ellos desarrollan significados subjetivos de sus experiencias" (Creswell, 2003, p. 19), la expectativa es dar voz a los docentes que transitan en los procesos evaluativos de acuerdo a sus vivencias y les otorgan un sentido que relacionan directamente con su vida laboral.

Con un enfoque cualitativo, ya que "se basan más en una lógica y un proceso inductivo (explorar y describir, luego generar perspectivas teóricas). Van de lo particular a lo general" (Hernández, Fernández y Baptista, 2014, p. 8), se indaga en diferentes fuentes de información para conocer, vincular y contrastar las diversas directrices que toma la investigación, por lo que no es su propósito generalizar una situación en cualquier contexto, más bien interpretarla y, en algunos casos, brindar opciones de solución a los fenómenos sociales. Bajo este enfoque, se reconstruye la realidad desde el punto de vista de quienes inciden en ella.

El método fenomenológico está inmerso en el trabajo que se presenta, porque "pone en primer plano de su atención los fines, la orientación del pensamiento y de la 
acción humana" (Bolio, 2012, p. 20), por las experiencias que los docentes evaluados compartieron a través de diversas entrevistas. Bolio (2012) menciona a Husserl (1990) al decir que la fenomenología "es una ciencia apriorística porque parte de la vivencia del sujeto, y la vivencia considerada como vivencia intencional... que no parte del objeto, sino de la conciencia de quien observa al objeto" (p. 23), esto es, la forma en que los individuos captan la intención de los hechos en su propio espacio y tiempo, lo que les permite decidir sus acciones en un futuro.

El instrumento que se utilizó para recabar la información fue un cuestionario que se aplicó a manera de entrevista, primero con un grupo focal conformado por cinco docentes y directivos que ejercen su profesión en el nivel educativo de primaria, además de otras cinco entrevistas individualizadas a directivos, asesor técnico pedagógico y docentes certificados en evaluación, mismos que han sido convocados por las autoridades educativas a revisiones en los procesos evaluativos a docentes en años anteriores.

Cabe destacar que se realizaron adecuaciones a los cuestionarios según el sujeto entrevistado y el rol que desempeña profesionalmente. El guión de entrevista se dividió en tres partes: el servicio docente que se presta, ámbito personal y evaluación estandarizada; para los docentes frente a grupo constó de 56 cuestionamientos, para directivos 43 y para evaluadores 30 . El análisis de resultados se obtuvo por medio del programa Atlas.ti, en el que se transcribieron los audios de las entrevistas que se realizaron, lo que facilitó triangular la información y la creación de redes que explican el hecho que acontece.

Para ayudar a explicar la problemática que se presenta, surge la pregunta principal: ¿Cuáles son las repercusiones de una evaluación estandarizada en el ámbito personal, en la experiencia de los docentes evaluados?

\section{RESULTADOS}

Mediante el análisis realizado con la información que arrojaron las entrevistas se determinaron redes categoriales que a continuación se presentan.

La figura 1 explica el sentir de muchos maestros hacia la evaluación; durante las entrevistas la palabra que usaron más para describirlo fue "punitiva", consideraron que se hizo con la intención de depurar a un gran porcentaje de maestros en servicio, opinan que las autoridades gubernamentales y educativas consideraron pertinente renovar el sistema con nuevos elementos que no necesariamente tuviesen formación normalista, por ende la forma adecuada fue mediante la evaluación como una herramienta de selección; por eso, lograron en su tiempo modificar las leyes para que este hecho se consumara.

Díaz (2019) alude a Arnaut (2015) al decir que se utilizó a la ley como arma de poder y control punitivo, al hacer a la evaluación obligatoria a costa del propio trabajo. Esta situación creó un ambiente de temor, los cambios legales se vivieron 


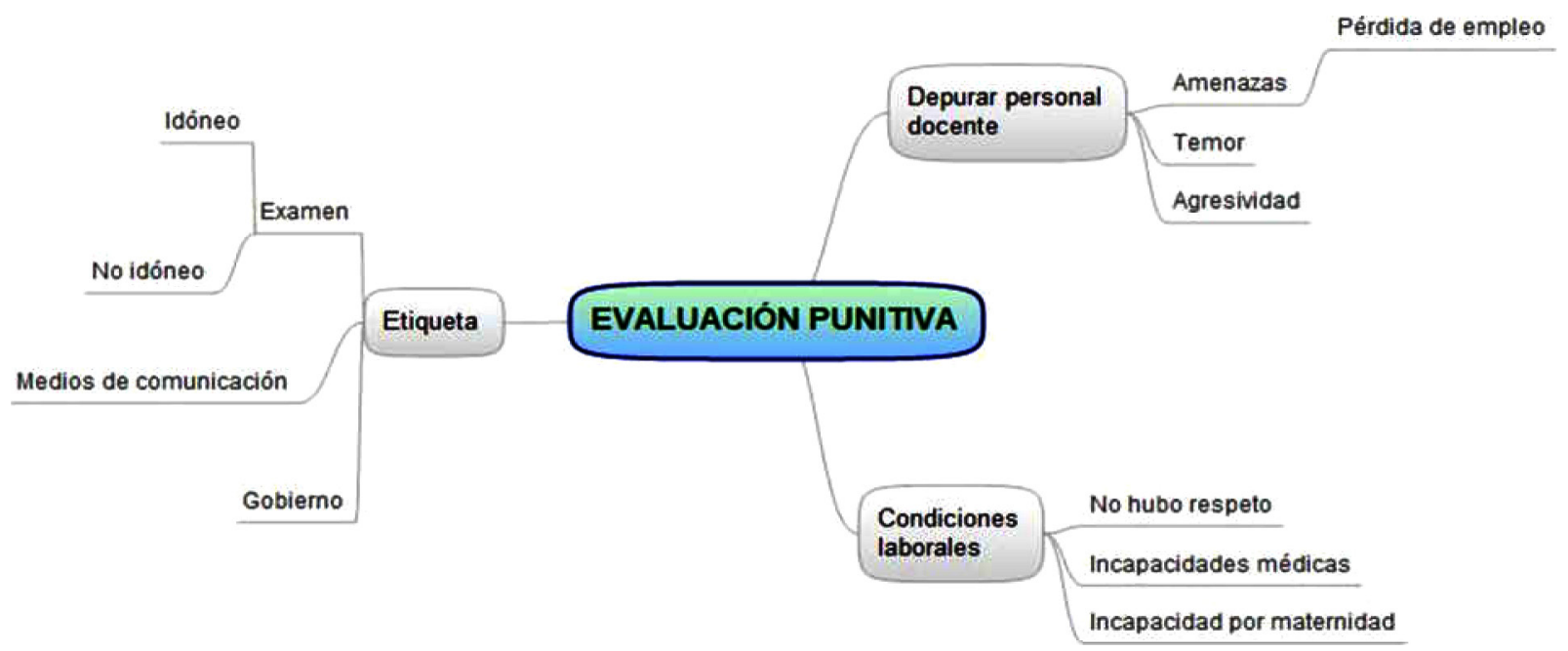

Figura 1. Evaluación punitiva.

Fuente: Elaboración propia.

de manera coercitiva, agresiva, se ponía en juego su trabajo, lo que a su vez desataría otras consecuencias aparte de la inestabilidad laboral, perder el sustento familiar, los años de servicio que no se tomarían en cuenta, así que, al ser de esta forma, el estrés aumentó sobremanera. Díaz comenta lo siguiente:

Los maestros después de vivir la experiencia de la evaluación adoptaron las siguientes figuras:

a) temor, ya que les hizo interiorizar miedo por las consecuencias; b) control, la vieron como instrumento para ser controlados socialmente, y c) sometimiento, pues les obligó [a] asumir esas actitudes de sumisión cediendo ante lo ineluctable [Díaz, 2019, p. 26].

Los docentes externaron que no se respetaron las situaciones laborales individuales, por ejemplo, había quien tenía licencia médica o por maternidad, y tuvieron la necesidad de presentar proyecto, evidencias, tareas evaluativas, diagnóstico, el examen; con el temor de perder el empleo si no acudían al llamado de las autoridades educativas.

Los docentes manifiestan que fueron etiquetados de acuerdo con los resultados de su evaluación, pero argumentan que no se consideraron las condiciones que se viven en cuanto a la institución en que se labora, el aula, el contexto, las personales, ya que no siempre son las óptimas para presentar el examen, sin embargo, es su deber profesional cumplir con lo que les es encomendado.

A pesar de esas situaciones se les etiquetó como "idóneo" o "no idóneo", como si de la simple etiqueta dependiera brindar un servicio de excelencia, pero al tomar como medida y referencia una evaluación estandarizada, homogénea, clasificó a los docentes si se es o no apto, lo cual los obligó a seguir profesionalizándose, pero bajo medidas restrictivas para quien no cumpla con los requerimientos, como si fuese moneda de cambio.

A todo esto se le agrega el ambiente que generan los medios de comunicación en contra de la labor del profesor, se desprestigia y desvalora su función, lo que se 


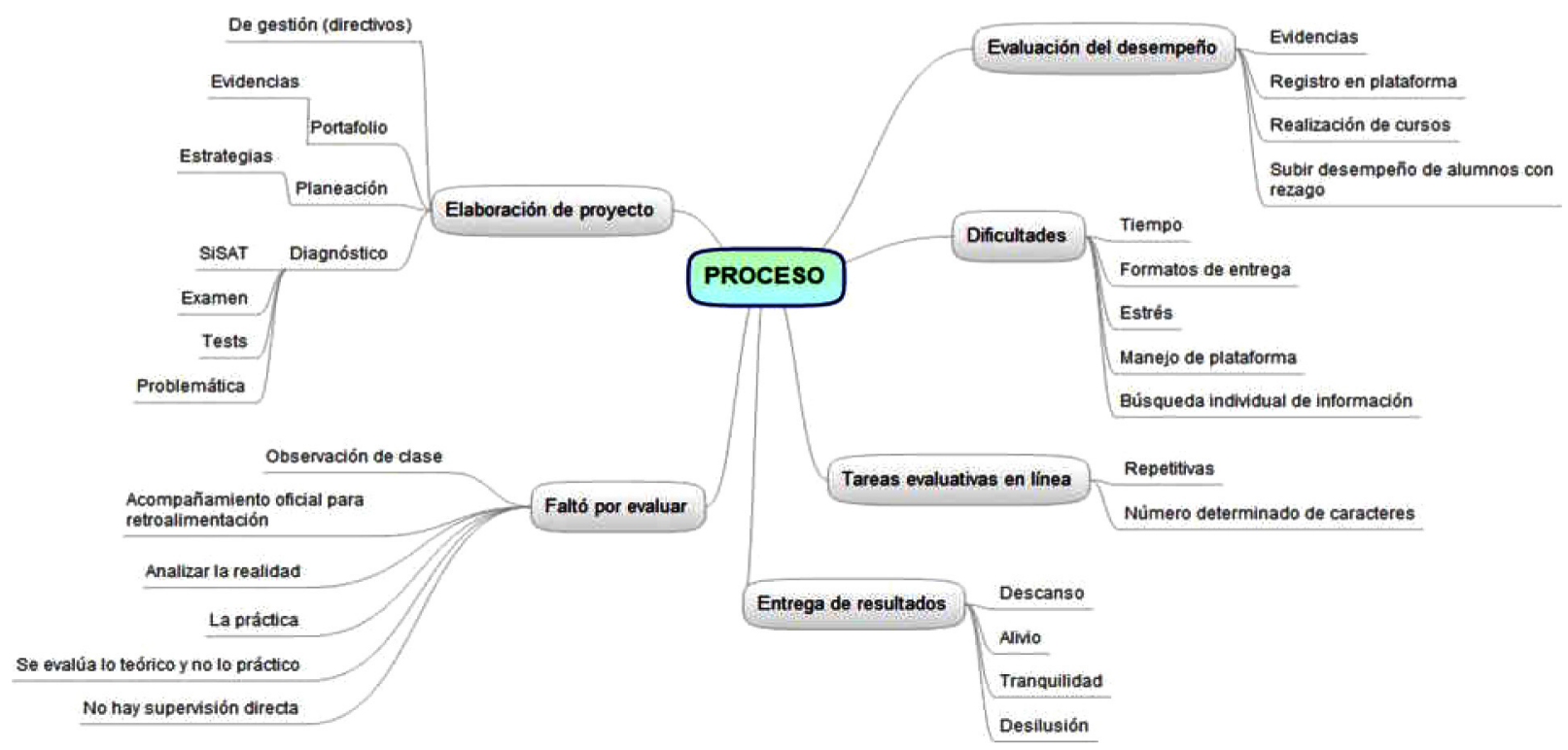

Figura 2. Proceso de evaluación docente.

Fuente: Elaboración propia.

refleja constantemente en el trato cotidiano con los padres de familia, que, como parte de la sociedad, desvirtúan su labor, incluso delante de los hijos. Lidiar con todo esto durante la evaluación, de cierta forma los desanimó.

En la figura 2 se expone el proceso que vivieron los docentes durante la evaluación, que se marcó por varios aspectos, entre ellos la elaboración de un proyecto: en el caso de los directivos fue uno de gestión; en los maestros de grupo, uno con base en las necesidades que se presentan en el aula; luego una planeación en la que atiendan esa problemática y las evidencias de esta.

Cabe mencionar que este procedimiento debiera hacerse siempre al comenzar el ciclo escolar, es el trabajo que le corresponde al maestro, pero cuando es para la evaluación, pareciera que es la primera vez que lo realizan, por la dificultad y la poca práctica que se tiene para su elaboración, lo que indica que, o no se cumple a cabalidad con las funciones que por normativa deben aplicarse, o se hacen solo por compromiso, sin que exista una reflexión hacia la práctica.

Una manera de evaluar el desempeño de los docentes en el proceso es a través de las evidencias que se suben a la plataforma, en la que se tienen que registrar previamente; de forma voluntaria hay que asistir a algún curso que se oferte para prepararse exclusivamente para la evaluación, puede tener o no costo económico, depende de las opciones que se presenten. Los maestros mencionan que las tareas evaluativas que tuvieron que registrar en línea fueron muy repetitivas, con cierto número de caracteres, lo que de cierta forma les entorpeció su redacción, tenían que buscar la forma de exponer sus puntos medulares sin parecer que decían lo mismo en un formato limitado. 
De entre las dificultades que mencionaron, la primera fue el tiempo limitado en la plataforma para subir evidencias y tareas evaluativas, argumentan que con una actividad o estrategia que aplicaron no siempre se ven los resultados favorables de forma inmediata, sin embargo tenían que mostrar que sí fueron exitosas; el estrés, la búsqueda de información, la poca habilidad digital para manejar la plataforma, también fueron conflictos que tuvieron que sortear, a veces sin ninguna ayuda. Por otro lado, la entrega de resultados fue para ellos motivo de alivio, tranquilidad y descanso, en algunos casos de desilusión por no obtener los resultados que se esperaban.

Saavedra y Saavedra (2014) emiten varias recomendaciones en los resultados de su investigación: proponen un proceso con base en el reconocimiento a la formación pedagógica; mencionan que la evaluación comienza cuando hay respeto al conocimiento, autonomía del docente; no perder de vista que el principal propósito de la educación es la formación del ser humano, no las pruebas o títulos de maestros o estudiantes, además, en el aspecto ético, cada profesor tiene sus propias cualidades, por lo que una prueba estandarizada no asegura tener la evidencia suficiente para medir sus saberes profesionales; un acercamiento cualitativo brindaría una cualificación del aspecto laboral, que le permita continuar con su formación y desarrollo.

En la opinión de los maestros, algo que faltó por evaluarles fue la práctica, en ningún momento tuvieron la supervisión de ninguna autoridad con la finalidad de enriquecer, denotar áreas de oportunidad y/o retroalimentarles; nunca asistió nadie a observarlos, por lo que el sentimiento de verse solos fue constante, así que la única ayuda que tuvieron fue la de los cursos y otros docentes que vivían la misma situación; en algunos casos sí hubo la asistencia de asesores técnicos pedagógicos y supervisores, pero siempre en horarios externos a los oficiales.

Fernández, Alcaraz y Sola consideran que la evaluación estandarizada puede ser útil cuando el propósito sea analizar los progresos de escuelas o sistemas educativos, pero no es eficaz para determinar prácticas de mejora, porque no se observan los procesos ni los factores que tienen que ver con el desarrollo, habilidades y destrezas de quienes son evaluados, se toman en cuenta como sujetos pasivos; además las condiciones en las que las que presentan no siempre son las mismas ni se ajustan a la realidad que viven diariamente. Por consiguiente, con dificultad se miden aprendizajes reales, puesto que no se puede tener un control exacto de las estructuras cognitivas de los sujetos, a lo que refieren: "si bien estas pruebas diagnósticas están bien realizadas y todos los años se va mejorando su diseño, siguen en su mayor parte buscando la competencia reproductora” (Fernández, Alcaraz y Sola, 2017, p. 56).

En la figura 3, con referencia al ámbito personal del docente que se enfrenta a una evaluación, se pueden denotar cuatro aspectos principales que le afectan de manera considerable. El primer punto son las condiciones laborales en las que se dio esta evaluación, ya que externaron que fue injusto que no se respetaran los derechos de quienes tenían una incapacidad médica o por maternidad, y fueran obligados a 


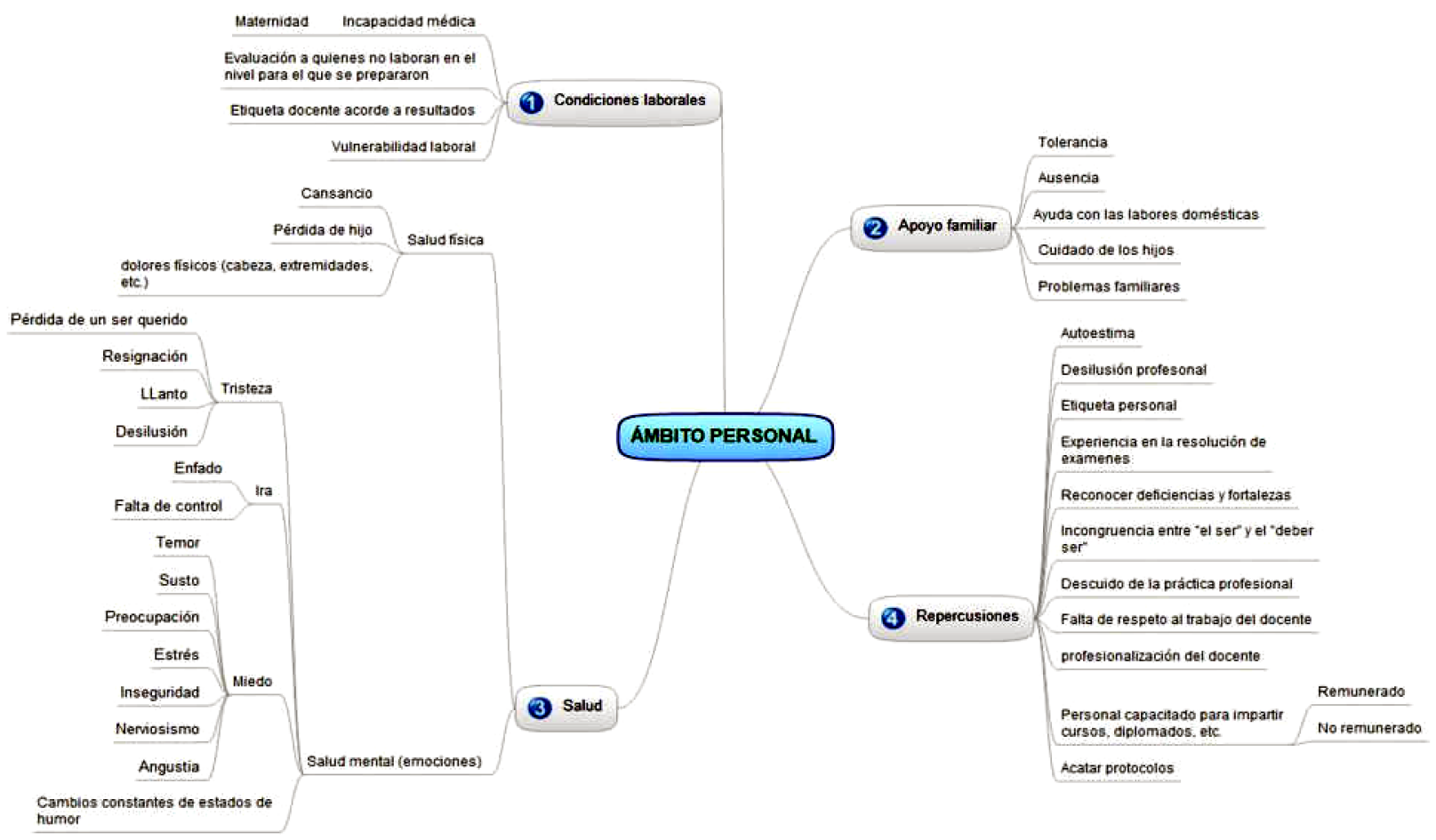

Figura 3. Ámbito personal del docente evaluado.

Fuente: Elaboración propia.

presentar el examen y mandar sus evidencias por medio de la plataforma creada para ello, sin importar sus condiciones o estado.

Hubo también el caso de docentes que no trabajan en el nivel educativo para el que se prepararon y se les convocó a evaluarse, por ejemplo, hay quienes son profesores de preescolar y laboran en educación primaria, hay quienes estudiaron la profesión de Educación Física y trabajan en preescolar o primaria como titulares de grupo. En su experiencia, consideran que, debido a esta situación, ser evaluados fue un pretexto para depurar personal; cabe mencionar que las mismas autoridades educativas los contrataron para ocupar esos puestos por necesidades del sistema, sin brindarles una preparación previa para lo que tenían que enfrentar, lo que les obligó a prepararse a marchas forzadas o conforme se presentaran las situaciones propias del oficio.

Aunado a esto, verse desprotegidos en caso de que sus resultados no fueran satisfactorios y cargar profesionalmente con una etiqueta, aún con varios años de antigüedad en el servicio, dan como resultado sentirse vulnerables al estar en manos de alguien más con la autoridad de decidir, y para lo cual no hay excusas ni reclamos, ya que el veredicto es inapelable. Así que, ser etiquetados de esta manera afectó negativamente a quienes de alguna forma no completaron el puntaje que se solicitó para aprobar.

El segundo punto, referente al papel que jugó la familia, fue trascendente, la mayoría de los profesores manifestaron el apoyo incondicional de parte de todos 
sus miembros para brindarles el tiempo suficiente y que pudieran completar sus tareas evaluativas, ordenar y enviar evidencias. El tercer punto, referente a la salud, se divide en dos aspectos, el físico y el mental; en el primero, los maestros externan diferentes tipos de dolencias físicas y cansancio, incluso el caso de la pérdida de un embarazo en pleno examen, como consecuencia del estrés que se vivió. En el mental, hacen alusión a las emociones básicas y sus consecuencias, como el enfado, temor, inseguridad, nerviosismo, etc. No se mencionó la felicidad, al parecer no agradó a ningún docente recibir la noticia de que serían evaluados.

Algunas repercusiones que vivieron los maestros en este ámbito fueron de desilusión profesional al no obtener los resultados para los que consideran que se prepararon, su autoestima bajó por la misma causa, o en otros casos, aunque aprobaron satisfactoriamente, les afectó negativamente al no lograr el estímulo económico. La etiqueta profesional de esta evaluación logró mover en los docentes la percepción propia de su desempeño, que de alguna manera se manifestó en el trabajo diario dentro de las aulas; pero en el proceso se pudo constatar por viva voz de quienes lo transitaron que su práctica docente quedó de lado al momento de tener que documentarse e investigar lo referente al examen, al estar a cargo de su propia preparación, se descuidó su labor.

También externan que hubo incongruencias entre el ser y el deber ser como profesionales en la práctica, esto es, la normativa marca ciertas líneas a seguir, pero la realidad es otra, o al menos las reacciones y actitudes que demuestran son parte de su sentido común, y pudieron contrastarlas al tener conocimiento de lo que las reglas establecen, por lo tanto, al desconocer los procedimientos ya regulados, no se justifican acciones propias ajenas a lo que se solicita, por lo que, de cierta forma, el maestro se siente acorralado al cuestionarse a sí mismo. Lo efectivo en este caso es que, al conocer normas y protocolos, de cierta forma se está más que obligado a cumplirlo.

Ramos habla de un currículo moldeable que va acorde con las características propias de cada aula, como resultado creativo de quienes lo ejecutan, por lo tanto la evaluación no puede ser idéntica en todos los sentidos, "es inadecuado efectuar tal tipo de mediciones con una misma medida, en especial si se basan en modelos rígidos que descontextualicen la labor educativa y soslayen la interpretación de los fenómenos" (Ramos, 2020, p. 5); por consiguiente, el trabajo de un docente es complejo porque se robustece con la participación de muchos actores, así que no puede ser su responsabilidad exclusiva buscar la excelencia educativa.

Algo que afectó sobremanera a la profesión es la falta de respeto hacia los docentes, debido a los mensajes que se emiten en los diversos medios de comunicación, donde se desvalora y desvirtúa el trabajo de quienes se encuentran inmersos en este sistema, así que la evaluación resultó ser un instrumento del que pudieron echar mano para continuar con esta campaña de desprestigio, y, de ser una carrera prometedora, con bastante matrícula en sus escuelas de formación, pasó a casi ser anulada del 
currículo nacional, abriendo el panorama a otras profesiones afines que se pudieran desempeñar con más eficacia y eficiencia. Entonces, aquellos a quienes les tocó ser evaluados pudieron sentir de cierta forma el repudio de la sociedad en general.

Por otro lado, hubo repercusiones positivas, como la experiencia que adquirieron para contestar exámenes, al menos ya tienen una idea de cómo hacerlo; con base en sus resultados, al entregarlos de manera personal e intransferible, les permitió darse cuenta de sus fortalezas y áreas de oportunidad, el problema es que, para poder subsanarlas, tendría que ser de forma autónoma, ya que el sistema oferta cursos o diplomados que no siempre van encaminados a favorecerlas. Otro punto a favor es la necesidad que tuvo el docente de profesionalizarse, esto es, buscar ayuda pertinente de otros docentes más preparados, documentarse debidamente, reflexionar sobre su práctica y buscar alternativas de enseñanza diferentes a las propias para el logro de sus objetivos.

\section{DISCUSIÓN}

Respecto a la respuesta a la pregunta de investigación, “¿Cuáles son las repercusiones de una evaluación estandarizada en el ámbito personal, en la experiencia de los docentes evaluados?", se puede decir que transitar por este proceso tiene consecuencias negativas y positivas; en las negativas, los maestros entran a una situación de estrés extrema, lo que influye directamente en el aspecto familiar: no les brindan la atención esperada, disponen del tiempo efectivo con ellas para su preparación profesional, se generan discusiones a menudo y pueden llegar a una ruptura definitiva.

También se encuentra que la autoestima del docente disminuye al no sentirse capaz de aprobar el proceso, por lo que le cuesta más trabajo enfocarse en su preparación; esto le lleva a vivir en un estado casi permanente a la defensiva, creyendo que las autoridades educativas están en su contra, incluso compañeros de trabajo y la propia familia. El miedo a que le quiten o muevan su estado de confort le hace perder la confianza en sus propias habilidades y destrezas. La desilusión que acompaña la espera de los resultados de la evaluación incide directamente en hacer de esta experiencia algo negativo, difícil de superar. Respecto a la salud física, esta se afecta debido al estrés que se vive, y tiene como resultado alta presión arterial, dolores musculares, cefaleas, obesidad, desnutrición, urticaria, incluso, en el caso de mujeres embarazadas, la pérdida del bebé.

Existe a su vez la incongruencia de acuerdo a su sentido común entre el ser y el deber ser, ya que en la vida cotidiana de una institución educativa surgen diversas situaciones y problemas que conducen a los docentes a la toma de decisiones bajo sus propios riesgos, experiencias y sentido común, lo que no siempre concuerda con lo establecido en los protocolos y normativas a seguir, pero la causa es el desconocimiento que se tiene de las mismas, lo que no justifica acciones y errores que afectan directamente a otros actores educativos; la ignorancia en este rubro no exime 
la culpabilidad, porque es obligación del docente conocer y poner en práctica todo lo que acontece a su labor.

Otro aspecto negativo es la etiqueta personal que se asigna a quienes no han podido acreditar la evaluación; se les señalan sus áreas de oportunidad, pero no se atienden directamente por las autoridades educativas, lo que obliga al docente a trabajarlas de forma personal si es que tiene la iniciativa; si no fuese así, la consecuencia es que seguirá atendiendo a un puñado de alumnos baja una enseñanza deficiente.

Por otro lado, los docentes reconocen que al participar en la evaluación tuvieron que dejar de lado el trabajo continuo con su grupo, descuidar planeaciones semanales, estrategias diversas y acordes al interés de sus alumnos, etc. De cierta forma resulta incongruente, ya que se supone que elaborar un proyecto cuyo objetivo principal es la mejora de los aprendizajes debiera de ayudar a abatir el rezago, pero priorizaron "maquillar" actividades supuestamente implementadas y mostrar el lado bello de la situación, incluso por sugerencia de sus autoridades educativas. Se considera que desconocer la relación entre la teoría y la práctica es una gran barrera para considerar a la evaluación docente como un proceso aparte del hecho educativo.

Dentro de los aspectos positivos que deja la evaluación en el aspecto personal de los profesores se cuenta que, como lo mencionan de manera general, les enseña a buscar estrategias para poder responder a los diferentes cuestionamientos que se plantean en el examen de conocimientos y elaboración de productos y proyectos, lo que les hace más hábiles para procesos evaluativos posteriores.

La evaluación obliga a los maestros a investigar la forma de adquirir los conocimientos básicos para poder acreditarla, se incluyen en diversos cursos que se ofertan, crean grupos de autoayuda y estudio, buscan el apoyo de docentes más capaces o preparados; esto agrega más trabajo a sus horarios habituales, pero incide en su profesionalización, que, a la larga, otorga beneficios a todo el sistema educativo, ya que su enseñanza se transforma en afán de mejorar y perfeccionar su práctica.

Esta oportunidad genera espacios para los maestros más capacitados, que comparten sus saberes y conocimientos a quienes les solicitan el servicio, que puede ser o no remunerado, lo que crea redes extensas de apoyo en las que se socializan y disciernen los contenidos que generalmente brindan las guías de estudio cuando se emite una convocatoria para la evaluación. Es también en estos espacios, tanto individuales como grupales, que se exige a los profesores analizar la normativa que rige al sistema educativo, o sea, si a lo largo de su trayectoria laboral no han tenido la iniciativa o la oportunidad de conocerla, este proceso los fuerza a hacerlo y, por lo tanto, les exige ponerlas en práctica.

\section{CONCLUSIONES Y SUGERENCIAS}

Lo expuesto en este artículo es solo una pequeña parte de todas las dificultades que se tienen al aplicar una evaluación estandarizada a los docentes, y más cuando es 
obligatoria; las repercusiones en el ámbito personal marcan con experiencias no tan gratas a la mayoría de quienes han transitado por estos procesos. Actualmente, se realizaron ajustes legales para tomar en cuenta las necesidades del gremio magisterial, por lo que emanó de las autoridades educativas y gubernamentales la Ley General del Sistema para la Carrera de las Maestras y los Maestros, en la que se pretende dignificar la labor del maestro, se aclaran las funciones de cada actor educativo; dejan a la fecha solo la evaluación para la admisión y promoción, horizontal y vertical; quitan definitivamente la de permanencia, que fue la que más afectó a los profesores.

No cabe duda de que una evaluación es necesaria, los maestros externan que no están en contra de ser evaluados, pero sí de las condiciones en las que se hace; habría que continuar el análisis y ver la manera más factible de aplicarlo sin dañar los aspectos personales y profesionales de quienes se encuentran en el servicio. La experiencia que se vivió pudiera ser de gran ayuda para futuras recomendaciones acerca de los procesos y su pertinencia, lo que debiera incluir también, al menos, la observación y retroalimentación de lo que sucede dentro de las aulas.

Evaluar la práctica, darle seguimiento y acompañamiento por parte de las autoridades educativas, sería un aspecto clave para que los docentes tengan certidumbre al momento de ser evaluados, ya que con una asesoría pertinente se despejarían dudas y se fortalecería la enseñanza; al mismo tiempo, no sentirse solos en el proceso les brindaría seguridad para enfrentar el estrés. Se recomienda también una atención psicológica para los docentes más vulnerables, que conlleve el manejo asertivo de las emociones, el estrés y la autorregulación.

\section{REFERENCIAS}

Bolio, A. (2012). Husserl y la fenomenología trascendental: Perspectivas del sujeto en las ciencias del siglo XX. Reencuentro, (65), 20-29. Recuperado de: https://www.redalyc.org/ pdf/340/34024824004.pdf.

Creswell, J. (2003). Diseño de investigación. Enfoques cualitativo, cuantitativo y con métodos mixtos (2a. ed.). Lincoln: Universidad de Nebraska.

Cruz, Y., Hincapié, D., y Rodríguez, C. (2020). Apéndice de profesores a prueba: claves para una evaluación docente exitosa. Nota Técnica No. IDB-TN-1782. Banco Interamericano de Desarrollo.

Díaz, F. (2019). La evaluación obligatoria y su sentido para el profesorado de telesecundarias: entre el temor, control y sometimiento. Revista Iberoamericana para la Investigación y el Desarrollo Educativo, 9(18).

Fernández, M., Alcaraz, N., y Sola, M. (2017). Evaluación y pruebas estandarizadas: una reflexión sobre el sentido, utilidad y efectos de estas pruebas en el campo educativo. Revista Iberoamericana de Evaluación Educativa, 10(1), 51-67. DOI: https://doi.org/10.15366/ riee2017.10.1.003.

Hernández, F., Beltrán, J., y Marrero, A. (2011). Teorías sobre la sociedad, familia y educación (2a. ed.). España: Universidad de Valencia.

Hernández, R., Fernández, C., y Baptista, M. (2014). Metodología de la investigación (6a. ed.). México: McGraw-Hill Interamericana. 
Ramos, L. (2020). El docente como profesional de la educación. Artículo Editorial. Archivos en Medicina Familiar, 22(1), 1-6.

Saavedra, L., y Saavedra, S. (2014). El sentido pedagógico de la evaluación del profesorado. Sophia, (10).

SEP [Secretaría de Educación Pública] (2019). La Nueva Escuela Mexicana: principios y orientaciones. México: Subsecretaría de Educación Media Superior. Recuperado de: https://dfa. edomex.gob.mx/sites/dfa.edomex.gob.mx/files/files/NEM\%20principios\%20y\%20 orientacio $\% \mathrm{C} 3 \% \mathrm{ADn} \% 20$ pedago $\% \mathrm{C} 3 \%$ ADgica.pdf. 